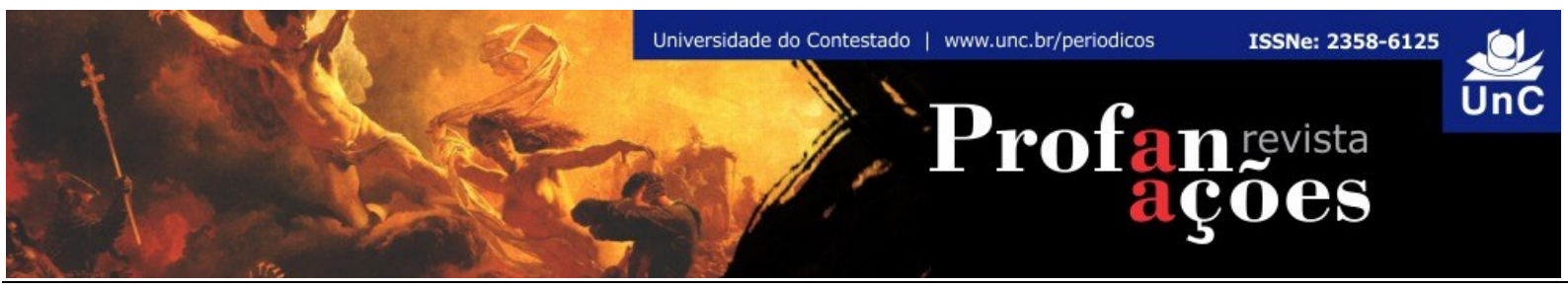

\title{
CARL SCHMITT, GIORGIO AGAMBEN Y LA ESTRUCTURA BIPOLAR DE LO POLÍTICO
}

Luciano Nosetto 1

RESUMEN: Este artículo parte de la constatación de que tanto Carl Schmitt como Giorgio Agamben conciben lo político como un juego de tensiones entre dos polos. Esta caracterización panorámica exige sin embargo una aproximación más pormenorizada. En esta línea, se procede en primer lugar a reconstruir la bipolaridad presente en la arqueología de la política desplegada en la saga Homo sacer, para luego identificar la recepción crítica que Agamben hace del pensamiento político de Schmitt. Seguidamente, se identifica la bipolaridad actuante en la forma política, tal como concebida por Schmitt. Hecho esto, se evalúa la razonabilidad de las críticas de Agamben, atendiendo especialmente a los escritos schmittianos contemporáneos al ascenso del nazismo. Finalmente, se establecen algunas conclusiones en lo relativo al tiempo histórico, a la teoría del Estado y a la práctica política contemporánea.

Palabras clave: Maquina política. Forma política. Teología política. Estado de excepción. Katéchon.

\section{CARL SCHMITT, GIORGIO AGAMBEN, AND THE BIPOLAR STRUCTURE OF THE POLITICAL}

ABSTRACT: This article opens with the assertion that Carl Schmitt and Giorgio Agamben share a common conceptualization of the political, defined as a tense interplay of two poles. This general assertion requires nonetheless a more detailed approach. Consequently we proceed in the first place to a reconstruction of the bipolarity present in the archeology of the political developed in the Homo sacer saga. Secondly, we identify Agamben's critical reception of Carl Schmitt's work. In the third place, we account for the bipolarity that characterizes Schmitt's political form. We later evaluate the reasonableness of Agamben's criticism, especially taking into account the Schmittian interventions during the rise of Nazism. Finally, conclusions are drawn in relation to the historical time, the theory of state, and contemporary political practice.

Keywords: Political Machine, Political Form, Political Theology, State of Exception, Katéchon.

\footnotetext{
${ }^{1}$ Doctor en Ciencias Sociales, Universidad de Buenos Aires. Investigador Adjunto del Consejo Nacional de Investigaciones Científicas y Técnicas (CONICET) con asiento en el Instituto de Investigaciones "Gino Germani" de la Facultad de Ciencias Sociales de la Universidad de Buenos Aires. Argentina. E-mail: Inosetto@gmail.com
} 


\section{INTRODUCCIÓN}

En el curso de sus reflexiones sobre la guerra civil, Giorgio Agamben se detiene en la promiscuidad de los términos polis y oikos. Siguiendo los estudios de Nicole Loreaux sobre la noción griega de stasis, señala Agamben que, durante la guerra civil, los vínculos políticos alcanzan una cercanía y solidaridad propias de la familia, mientras que la casa se ve atravesada por las líneas de adversidad propias de la política. Así, el locus de la guerra civil no es ni la ciudad ni la familia, sino aquel umbral en el que ambas se entrecruzan. Coronando estas reflexiones sobre la stasis, postula Agamben: "En la Grecia clásica al igual que hoy, no existe algo así como una política: la política es un campo incesantemente recorrido por las corrientes de tensión de la politización y la despolitización" (AGAMBEN, 2015, p. 30).

Con estas consideraciones, Agamben pareciera volver sobre los pasos de Carl Schmitt en su conceptualización de lo político. Al igual que Agamben, Schmitt se rehúsa a identificar una sustancia política, que permita confinarla a un locus propio o específico. Al igual que Agamben, Schmitt comprende lo político como un juego entre dos polos: la máxima tensión en la separación respecto de un otro, que es definido como enemigo, permite la neutralización del conflicto al interior del nosotros y la pacificación de la relación entre los amigos (SCHMITT, 2009, p. 25-26, 36, 6768; LALEFF ILIEFF, 2016, p. 63). De este modo, tanto Schmitt como Agamben inscriben lo político al interior de una estructura bipolar, definida por el juego entre politización y despolitización; juego que tiene por efecto la permanente producción de los polos de lo político y lo impolítico.

Así y todo, no es Schmitt el espejo en que Agamben quisiera verse reflejado. En una entrevista realizada en ocasión de la publicación de su libro sobre el estado de excepción, Flavia Costa consultó a Agamben sobre su relación con Schmitt. La respuesta de Agamben:

Era evidente (creo que es evidente para cualquiera que no sea estúpido ni tenga mala fe, o, como sucede a menudo, las dos cosas juntas) que si quería trabajar con el derecho y sobre la política, era con él con quien debía medirme. Como con un enemigo, desde ya -pero la antinomia amigoenemigo era precisamente una de las tesis schmittianas que quería poner en cuestión (AGAMBEN, 2004, p. 11). 
La respuesta de Agamben resulta ambivalente en más de un sentido. Ambivalente porque concede a Schmitt la estatura intelectual de un pensador ineludible al tiempo que lo reconoce como un evidente enemigo. Ambivalente también porque la vehemencia inicial de su respuesta (rayana en la indignación) se combina con un remate elusivo y confuso. Lo que no queda claro es cuál sería el sentido no schmittiano de la enemistad que Agamben cultiva contra Schmitt.

Una mirada panorámica de la obra de Agamben da cuenta de que, cualesquiera sean las razones de esa hostilidad, lo cierto es que el italiano se ha visto obligado a recorrer largo trecho en compañía del alemán. Es que los trabajos de Agamben y, en particular, su voluminoso proyecto de Homo sacer están sembrados de referencias a Schmitt; referencias que, en no pocos casos, resultan estructurantes de las ideas allí desplegadas (RAFFIN, 2015; TACCETTA, 2012, p. 47). La situación se asemeja a la de aquellos westerns en los que dos vaqueros enemistados se ven forzados a emprender un largo viaje juntos. La hostilidad es permanente; la solidaridad, inevitable.

Visto más de cerca, el camino recorrido en compañía de Schmitt tiene un final abrupto. Es que la presencia de Schmitt es central en los primeros episodios de Homo sacer, pero se enrarece a medida que la obra avanza. Este movimiento puede brindar una clave de inteligibilidad de la relación entre ambos. Veamos. Según describe Agamben, el proyecto Homo sacer consiste en una arqueología de la biopolítica, esto es, una indagación en torno a la estructura originaria de la política occidental, que articula de manera fundamental la política con la vida (AGAMBEN, 2014, p. 333). A un primer volumen, en que se presentan las premisas fundamentales de la investigación (El poder soberano y la nuda vida), sigue una segunda parte, compuesta de cinco tomos que proveen una serie de abordajes arqueológicos sobre el estado de excepción (Estado de excepción), la guerra civil (Stasis), el juramento (El sacramento del lenguaje), la economía (El reino y la gloria) y el oficio (Opus Dei). Seguidamente, la tercera parte ofrece una reflexión sobre la subjetividad ética a partir de la figura del testigo (Lo que queda de Auschwitz). La investigación adquiere finalmente un tono propositivo, señalando en las nociones de forma-de-vida (Altísima pobreza) y de uso (El uso de los cuerpos) las posibilidades de una nueva política. 
Premisas, diagnóstico, ética, política: con esta estructura en mente, puede aclararse la índole de la deuda de Agamben con Schmitt. La presencia del alemán es central en la primera parte de Homo sacer, allí donde Agamben presenta las premisas fundamentales de su indagación en torno la política occidental. Lo mismo puede decirse respecto de aquellos tomos de la segunda parte en los que Agamben se embarca en una arqueología del estado de excepción y de la guerra civil. En estos casos, Schmitt constituye una fuente de consulta privilegiada, que sirve de respaldo para las tesis agambenianas. Esto no implica una aceptación de la teoría schmittiana en bloque: más bien, Agamben se ve reiteradamente obligado a precisarla allí donde resulta demasiado general (1995, p. 24; 2015, p. 74-75), a completarla allí donde ofrece reconstrucciones unilaterales (1995, p. 15, 103; 2015, p. 27) y a actualizarla allí donde no captura los desarrollos más recientes (1995, p. 15-16). Como quiera sea, puede decirse que el diálogo con Schmitt es, en estos casos, esencial a la línea argumental de Homo sacer. Ahora bien, cuando Agamben aborda su arqueología del juramento, las referencias a Schmitt simplemente desaparecen. El reencuentro se produce en El reino la gloria, que se presenta como un trabajo abiertamente anti-schmittiano (AGAMBEN 2008, p. 120). Tras este choque frontal, los senderos simplemente se bifurcan y Agamben recorre la segunda mitad de su camino dejando atrás a su ocasional compañero. Si nuestra reconstrucción es correcta, deberíamos concluir que Agamben invita a Schmitt a formar parte de sus investigaciones arqueológicas sobre la política contemporánea, pero se separa de él en cuanto esas investigaciones dan lugar a reflexiones éticas y políticas de índole propositiva. La pars destruens de la saga se hace en compañía de Schmitt: en la pars construens, se lo deja de lado (MOYANO, 2017, p. 20). Esta doble disposición es compatible con la admiración que produce la estatura intelectual ineludible de Schmitt y el rechazo que genera su adhesión al nacionalsocialismo.

En lo que sigue, proponemos recorrer el camino compartido por Agamben y Schmitt y comprender las razones de su alejamiento. A estos efectos, comenzaremos reconstruyendo la estructura originaria de la política occidental, tal como mentada por Agamben, e identificaremos seguidamente cuál es el rol que desempeña Schmitt en este desarrollo. Hecho esto, consultaremos la obra del propio Schmitt, a efectos de evaluar la razonabilidad de las acusaciones agambenianas. 
Habiendo escuchado a ambas partes, estaremos finalmente en condiciones de reconstruir los términos del conflicto y de comprender qué las vuelve irreconciliables.

\section{LA MÁQUINA AGAMBENIANA: BIPOLARIDAD Y CENTRO VACÍO}

En el último volumen de su Homo sacer, Agamben ofrece una vista panorámica de la obra que está concluyendo. Allí explica que el proyecto de una arqueología de la política consistió precisamente en traer a la luz la estructura originaria de la política occidental. Sostiene Agamben que esa estructura "consiste en una ex-ceptio, en una exclusión inclusiva de la vida humana en la forma de la nuda vida". De allí que el "primer acto" de su investigación haya consistido en "la identificación de la nuda vida como el referente primero y la puesta en juego de la política" (AGAMBEN, 2014, p. 333).

Con esto, se describen las premisas fundamentales de su obra, contenidas en el primer volumen de Homo Sacer: El poder soberano y la nuda vida. Allí Agamben recuerda que la política occidental se asienta sobre la distinción clásica entre el oikos (ámbito de reproducción de la vida natural o de la zoé) y la polis (ámbito de desarrollo de la vida calificada o del bíos). De este modo, la política se funda sobre una cesura de la vida, que opera la distinción entre bíos y zoé, para incluir a la primera en el espacio público y confinar la segunda al ámbito doméstico. Ahora bien, si solo hay política sobre la base de la exclusión de la zoé, esto es decir que solo hay política allí donde se renueva incesantemente la cesura que distingue la vida calificada respecto de la vida no calificada. Por ende, el arcano de la política, su fundamento más íntimo, consiste en la renovada producción de esa vida no calificada o desnuda. Que lo más íntimo sea al mismo tiempo lo que debe excluirse define precisamente la estructura de la ex-ceptio: de aquello que es "capturado fuera" o incluido en la forma de su exclusión. De allí que "el carácter específico de la producción biopolítica no se[a] tanto la gestación de formas de vida determinadas [...] sino el aislamiento de un algo en los hombres que debe ser gestionado: la vida desnuda" (MOYANO, 2017, p. 16). En suma, la distinción apolínea entre los pares oikos/zoé y polis/bíos pierde de vista el hecho de que la nuda vida es aquello sobre cuya exclusión se funda la ciudad de los hombres. Al respecto concluye Agamben que "la estrategia es siempre la misma: algo se divide, se excluye y se expulsa al 
fondo $\mathrm{y}$, precisamente a través de esta exclusión, resulta incluido como arché y fundamento" (AGAMBEN, 2014, p. 334). Concretamente, "lo que está en juego en la relación entre oikos y polis es la constitución de un umbral de indiferencia en el cual lo político y lo impolítico, el afuera y el adentro coinciden" (AGAMBEN 2015, p. 30). Es este umbral, en que el orden jurídico-institucional entra en contacto directo con la nuda vida, el que da a ver que la biopolítica constituye el arcano del poder en Occidente.

Sobre la base de una misma estructura, Agamben presenta en su libro Stasis una arqueología de la guerra civil. Allí se ofrece un diagrama de la excepción que permite ganar claridad sobre el conjunto de la obra. Tal como mencionamos más arriba, Agamben identifica que la guerra civil confunde los ámbitos de la ciudad y la familia: es que, durante la stasis, los vínculos políticos alcanzan la intensidad de los lazos familiares, mientras que la unión familiar se ve cuestionada por las tensiones políticas. De este modo, la guerra civil reproduce la estructura de la excepción, que Agamben ilustra con el siguiente diagrama (AGAMBEN 2015, p. 30):

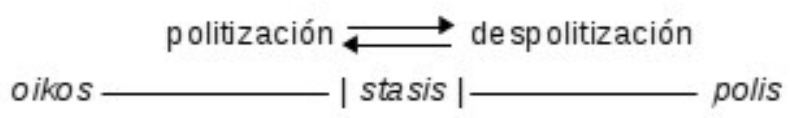

Intentemos comprender lo que da a ver este diagrama. Por un lado, la política se constituye a partir de un doble movimiento de politización de la ciudad y despolitización de la casa. Este movimiento centrífugo, que constituye los polos del oikos y la polis abre al mismo tiempo un hiato en el centro, dejando un espacio vacante. En ese centro vacío se produce la excepcional contaminación entre los términos opuestos; contaminación que, en este caso, tiene la forma de la stasis: de la guerra civil que confunde casa y familia, al punto de volverlas indistinguibles.

Este funcionamiento, que combina fuerzas centrífugas y polarizantes, ha sido magistralmente descrito por Germán Prósperi en su caracterización de la "máquina elíptica" agambeniana. Según Prósperi, Agamben combina la máquina circular de Furio Jesi, que funciona a partir de la remisión a un centro, con la bipolaridad postulada por Aby Warburg en su caracterización de la cultura occidental. Al introducir dos polos, la figura circular se comba, formando una elipse. Así, "el gesto propio de Agamben consiste en transformar la máquina circular de Jesi en una 
máquina elíptica, es decir, en una máquina que no sólo se define a partir de un centro, sino también a partir de dos focos" (PRÓSPERI, 2015, p. 73). Andrea Cavalletti ha señalado también la influencia de Furio Jesi en la concepción agambeniana de la máquina política, pero ha reemplazado a Aby Warburg por Carl Schmitt: "el gesto resolutivo de Agamben [...] consiste quizás en el acercamiento, más bien, en la combinación de la máquina -mecanismo que 'pone su origen en el afuera de sí que es su interior más remoto'- con otro modelo [...], de ascendencia en cambio schmittiana, el modelo de la 'excepción”' (CAVALLETTI, 2010, p. 54).

Como quiera sea, si asumimos que, con esta disposición gráfica de la guerra civil, Agamben está dando a ver la estructura general de la excepción, resulta entonces posible traducir los diferentes episodios de Homo sacer a este mismo esquema. En tal caso, la relación entre bíos y zoé que repusimos más arriba podría aclararse mediante el siguiente diagrama:

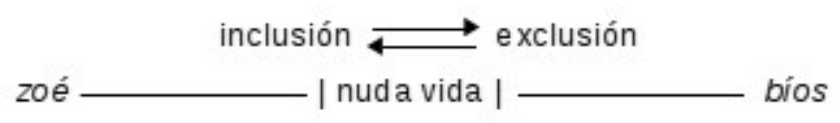

Esto da a ver que la política occidental se funda sobre una cesura en el continuum de la vida, que distingue la vida calificada respecto de la vida no calificada, y las aparta en un doble movimiento de inclusión del bíos en la ciudad y reclusión de la zoé a la casa. Este movimiento centrífugo abre un centro vacío e indeterminado, en el cual los términos opuestos se confunden y se vuelven indiscernibles. En ese umbral de indistinción, zoé y polis se encuentran sin mediaciones, produciendo la excepcional politización de la nuda vida.

Este mismo esquema debería permitirnos ilustrar el estado de excepción abordado en el primer tomo de Homo sacer II. Recuperando los desarrollos de Carl Schmitt, Agamben postula que el orden jurídico se erige sobre la exclusión de la anomia. Pero, en situaciones extremas, la conservación del orden jurídico puede exigir cursos de acción reñidos con la norma vigente. En tales casos, los poderes constituidos deben abrir paso a una autoridad que pueda actuar por fuera de la ley a efectos de hacer lo necesario para restituirla. Esta paradójica estructura de la excepción puede esquematizarse del siguiente modo: 


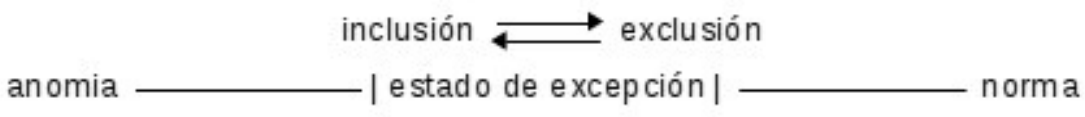

Aquí puede verse cómo la máquina jurídico-política, que vincula norma y poder, se funda sobre la exclusión de la anomia, esto es, de la autoridad sin ley. Sin embargo, en el centro de la máquina se produce un umbral de indistinción en el que la autoridad suspende la norma para restituir su vigencia. Al respecto, Agamben compendia:

\begin{abstract}
En Estado de excepción, la máquina jurídico-política de Occidente había sido descrita como una estructura doble, formada por dos elementos heterogéneos y sin embargo, íntimamente coordinados: uno normativo y jurídico en sentido estricto (la potestas) y uno anómico y extrajurídico (la auctoritas). El elemento jurídico-normativo, en el que parece residir el poder en su forma eficaz, tiene sin embargo necesidad de aquel elemento anómico para poderse aplicar a la vida; por otra parte, la auctoritas puede afirmarse y tener sentido solo en relación a la potestas. El estado de excepción es el dispositivo que debe, en último análisis, articular y tener juntos los dos aspectos de la máquina jurídico-política, estableciendo un umbral de indecibilidad entre anomia y nomos, entre vida y derecho, entre auctoritas y potestas (AGAMBEN, 2014, pp. 334-335).
\end{abstract}

Finalmente, Agamben reconoce que una estructura análoga está en juego en su arqueología de la economía, desplegada en El reino y la gloria. En este volumen, Agamben afirma que "la doble estructura de la máquina gubernamental, que en Estado de excepción aparecía en la correlación entre auctoritas y potestas, toma aquí la forma de la articulación entre Reino y Gobierno" (AGAMBEN, 2007, p. 9-10). Si el gobierno remite al poder como gestión eficaz, el reino alude a la majestuosidad litúrgica. Esto da lugar a una máquina gubernamental en la que el Rey queda excluido del gobierno y confinado al ceremonial, mientras que el gobierno despliega una incesante operatoria, que lo aleja de toda ociosidad. Sobre la base de estos términos polarizados, sostiene Agamben que el gobierno no puede operar sin remitir a esa majestuosidad que había sido primeramente excluida. De este modo, la inoperosidad es recapturada en la forma de la gloria, es decir, de aquella "zona incierta en la que se mueven aclamaciones, ceremonias, liturgia e insignias" (AGAMBEN, 2007, p. 209). 


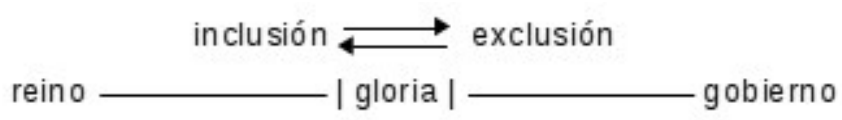

De este modo, la máquina gubernamental funciona sobre la base de la exclusión de la ociosidad, que queda confinada al ámbito del reino; pero esa ociosidad es recapturada en la forma de gloria que, en nuestras sociedades del espectáculo, resulta el rasgo central de la política democrática. Compendia Agamben que "el Gobierno glorifica el Reino y el Reino glorifica el Gobierno. Pero el centro de la máquina está vacío y la gloria no es más que el esplendor que emana de aquel vacío" (AGAMBEN, 2008, p. 233-234).

En suma, siguiendo el hilo de las analogías estructurales postuladas por Agamben, es posible dar a ver la estructura fundamental de la política. Se trata de un dispositivo que parte del establecimiento de una cesura entre lo que debe quedar dentro y lo que debe ser excluido, produciendo la distinción entre lo político y lo impolítico en sus diversas configuraciones: polis/oikos, bíos/zoé, norma/anomia, potestas/auctoritas o gobierno/reino. Al empujar ambos términos hacia sus polos, la máquina política abre un hiato en su mismo centro, que funge de umbral de indistinción entre lo político y lo impolítico.

\footnotetext{
En todas estas figuras opera un mismo mecanismo: el arché se constituye escindiendo la experiencia fáctica y expulsando al origen -es decir, excluyendo- una mitad de sí, para después rearticularla con la otra, incluyéndola como fundamento. Así, la ciudad se funda sobre la escisión de la vida en nuda vida y vida políticamente calificada; el humano se define a través de la exclusión-inclusión del animal; la ley, a través de la exceptio de la anomia; el gobierno, a través de la exclusión de la inoperosidad y su captura en la forma de la gloria (AGAMBEN, 2014, p. 336).
}

En suma, la reconstrucción de la estructura originaria de la política occidental, tal como mentada por Agamben, permite describir el funcionamiento de la máquina política en sus diferentes declinaciones. Es momento de identificar cuál es el rol que le cabe a Carl Schmitt en este recorrido. 


\section{LA EXTRAPOLACIÓN MODERNA}

Hemos mencionado la ambivalencia que despierta Carl Schmitt al interior del proyecto de Homo sacer. Habiendo repuesto la comprensión agambeniana de la estructura originaria de la política, estamos en mejores condiciones de precisar esa ambivalencia.

A decir verdad, Schmitt produce en Homo sacer no dos sino tres reacciones diversas: por un lado, Agamben reconoce el aporte intelectual del alemán; por otro lado, señala el carácter unilateral e incompleto de su teoría; finalmente, denuncia su contribución a la conformación de una máquina letal.

El reconocimiento del carácter ineludible del legado schmittiano es ostensible. Ya hemos comentado la opinión de Agamben según la cual todo aquel que quiera estudiar seriamente la política y el derecho deberá medirse con la obra del alemán. Es Schmitt quien formula la definición de la soberanía que sirve de base a la arqueología agambeniana y quien expone con mayor claridad la paradoja inherente a este concepto (AGAMBEN, 1995, p. 15, 19). A ello se suma el hecho de que "el intento más riguroso de construir una teoría del estado de excepción es obra de Carl Schmitt" (AGAMBEN, 2003, p. 44). En sus estudios sobre la guerra civil, Agamben añade que, junto con Hannah Arendt, le debemos a Schmitt la introducción del concepto de "guerra civil mundial" (2015, p. 10). Finalmente, en El reino y la gloria, reconoce Agamben que Schmitt da testimonio de la pervivencia de la gloria en las democracias contemporáneas, bajo la forma de opinión pública (AGAMBEN, 2007, p. 278-279). Ahora bien, este frecuente reconocimiento de la importancia del legado schmittiano coexiste en Agamben con el señalamiento de sus limitaciones.

Precisamente, es habitual encontrar en Homo sacer menciones al carácter unilateral e incompleto de la perspectiva schmittiana. Tomemos el caso de El poder soberano y la nuda vida. Si bien allí el punto de partida viene dado por la definición schmittiana de soberanía, lo cierto es que, para Agamben, Schmitt "se limit[a] a identificar quién, en el interior del orden jurídico, est[á] investido de unos poderes determinados, sin que eso sup[onga] que el propio umbral del ordenamiento [sea] puesto en ningún momento en tela de juicio" (AGAMBEN, 1995, p. 15). En este caso, Schmitt permite abordar el orden jurídico-político, pero pierde de vista la imbricación con la vida que define de la biopolítica. 
Algo similar sostiene Agamben en su volumen sobre la stasis. Allí se recuerda que Schmitt define a la política a partir de una lógica amigo/enemigo que coloca al enemigo fuera de la unidad. Al respecto observa Agamben que, "si un cierto ámbito es definido por una pareja de conceptos opuestos, ninguno de los dos puede ser íntegramente excluido sin comprometer la realidad" (AGAMBEN, 2015, p. 27). El riesgo de la disociación schmittiana es el de olvidar la persistencia de la guerra civil al interior del orden. Finalmente, en su arqueología de la economía, Agamben también señala la unilateralidad schmittiana. Es que Schmitt habría privilegiado la perspectiva jurídico-estatal, soslayando la dimensión económico-gubernamental (AGAMBEN 2014, p. 82). Ya en Homo sacer I, Agamben anticipaba esta unilateridad schmittiana. Allí se sostenía que, si la soberanía remite desde Jean Bodin a una puissance absolue et perpétuelle, lo cierto es que Schmitt se habría limitado a estudiar el carácter absoluto de tal poder, perdiendo de vista la dimensión ceremonial y litúrgica asociada a su perpetuidad (AGAMBEN, 1995, p. 103).

En suma, la unilateralidad del alemán es manifiesta en el hecho de que Schmitt ha investigado la soberanía, pero perdió de vista su vinculación con la vida; ha investigado la enemistad, pero perdió de vista sus manifestaciones civiles; ha investigado el orden jurídico, pero perdió de vista la dimensión del gobierno. Si esta fuera la última palabra respecto del alemán, podríamos concluir que la relación de Agamben con Schmitt es la que se tiene con un insumo necesario pero insuficiente. Sin embargo, en varios episodios de Homo sacer, la consulta del corpus schmittiano abre paso a la confrontación abierta.

El primer desencuentro tiene lugar en el último capítulo de El poder soberano y la nuda vida, allí donde Agamben postula que el campo de concentración se ha convertido en el nomos de lo moderno. En línea con la tesis octava de filosofía de la historia de Walter Benjamin, afirma Agamben que, en la modernidad, la excepción se convierte en la regla. Esto implica que la politización de la nuda vida (que, desde la Grecia clásica, se encontraba contenida en aquel umbral excepcional de indeterminación) acaba por expandirse sobre la totalidad del campo político.

Ahora bien, este desarrollo contemporáneo en virtud del cual regla y excepción se vuelven indiscernibles debería desbaratar la teoría de la soberanía schmittiana, fundada precisamente sobre la estricta delimitación de la decisión sobre la excepción. Sin embargo, sostiene Agamben que Schmitt incorpora con 
entusiasmo estas novedades a su obra. Concretamente, Agamben describe que, en el texto Estado, movimiento, pueblo, de 1933, Schmitt celebra la incorporación en el orden jurídico de la cláusula de "igualdad de estirpe" [Artgleichheit], que funciona como una remisión intranormativa al orden concreto. Del mismo modo en que ciertas normas remiten a la moral, a las buenas costumbres o a los usos comerciales, el desarrollo del derecho nacionalsocialista ha incorporado a su articulado el concepto de raza. Agamben advierte que la introducción de estas categorías hace estallar la distinción entre lo normativo y lo fáctico, entre cuestiones de derecho y de hecho, produciendo la coincidencia inmediata entre lo político y lo impolítico y, en definitiva, entre el poder soberano y la nuda vida (AGAMBEN, 1995, p. 191-195).

Estas consideraciones son retomadas en el volumen agambeniano dedicado al estado de excepción. Según vimos, allí el funcionamiento de la máquina jurídicopolítica es descrito a partir de la distinción entre el elemento normativo de la potestas y el elemento anómico de la auctoritas. Si bien el orden jurídico supone la exclusión de la anomia, esta queda sin embargo incluida en la figura de la autoridad sin ley que se activa durante el estado de excepción. Agamben describe la dialéctica entre auctoritas y potestas a partir de sus antecedentes romano y medieval:

\begin{abstract}
En tanto los dos elementos permanecen correlativos, pero conceptualmente, temporalmente y subjetivamente distintos -como funcionaba en la Roma republicana la contraposición entre senado y pueblo, o la contraposición entre poder espiritual y poder temporal en la Europa medieval-, la dialéctica que se da entre ellos, aunque fundada sobre una ficción, puede con todo funcionar de algún modo. Pero cuando ellos tienden a coincidir en una sola persona, cuando el estado de excepción, en el cual ellos se ligan y se indeterminan, se convierte en la regla, entonces el sistema jurídico-político se transforma en una máquina letal (AGAMBEN 2003, p. 110).
\end{abstract}

La máquina jurídico-política se corrompe allí donde poder y autoridad coinciden en una misma persona. Es en las figuras del Duce y del Führer que se produce esa coincidencia. Retomando a Max Weber, Heinrich Triepel y Pietro de Francisci, señala Agamben que la Führung se define por una serie de atributos personales, originarios e intransferibles. Es Carl Schmitt quien más claramente identifica el componente vital del conductor, definido por "la identidad de estirpe entre el jefe y sus partidarios" (AGAMBEN 2003, p. 107). Así, "las cualidades de Duce o de Führer están inmediatamente ligadas a la persona física y pertenecen a la tradición biopolítica de la auctoritas y no a la jurídica de la potestas" (AGAMBEN, 2003, p. 
107). Cuando Mussolini y Hitler asuman el poder, estos componentes biopolíticos de la auctoritas eclipsarán la dimensión jurídica de la potestas, volviendo ambos términos indiscernibles (TACCETTA, 2012, p. 131, 155-156). Aquí nuevamente, Schmitt contribuye a plegar un polo sobre otro, transformando la máquina jurídicopolítica en una máquina letal.

Finalmente, Schmitt asume en El reino y la gloria un rol análogo. Si la máquina gubernamental venía definida por los polos del reino y el gobierno, entre los cuales se abría un centro vacío en que se localizaba la gloria, nuevamente en este caso, Schmitt contribuirá a expandir la indistinción entre ambos polos, volviendo regla lo que hasta entonces era excepción. Sostiene Agamben al respecto:

\footnotetext{
Desde el punto de vista teórico, el debate [es] entre "gubernamentalistas" (que conciben el poder como ya siempre articulado según una doble estructura: potestad y ejecución, Reino y Gobierno) y los partidarios de una soberanía de la que no es posible separar la potencia y el acto, ordinatio y executio (AGAMBEN, 2007, p. 118).
}

En este marco, Agamben señala la hostilidad schmittiana ante toda separación entre reino y gobierno (AGAMBEN, 2007, p. 89-90). Dada esta indistinción, Schmitt podrá expandir el elemento litúrgico de la aclamación al punto de la saturación de la máquina gubernamental. En la interpretación de Agamben, las críticas de Schmitt a la democracia liberal representativa lo llevarán a afirmar que el pueblo solo se manifiesta en la forma de la aclamación. Así "la estrategia de Schmitt es clara: mientras retoma [...] la función constitutiva de la aclamación litúrgica, se presenta como el teórico de la democracia pura o directa para contraponerla a la democracia liberal de Weimar" (AGAMBEN, 2007, p. 192). En su crítica al liberalismo, Schmitt anticipa la exacerbación de la acclamatio que definirá la liturgia nacionalsocialista.

Estamos ahora en condiciones de caracterizar el rol que Agamben asigna a Schmitt al interior de la saga Homo sacer. Como hemos visto, en un primer momento, Agamben reconoce que la obra de Schmitt resulta ineludible para toda investigación abocada a los conceptos de soberanía y excepción, pero señala también que ese legado debe ser integrado al interior de una perspectiva más cabal, que dé cuenta de aquello que Schmitt perdió de vista. Sin embargo, la relación con Schmitt se enrarece a medida que Agamben se acerca a sus textos contemporáneos 
a la crisis de Weimar y al ascenso del nazismo. En estos materiales, Agamben visualiza una serie de dislocaciones schmittianas que corrompen la estructura de la exceptio. Daría la impresión de que el carácter unilateral de la teoría schmittiana habría hecho mella en su capacidad de orientación política, conduciéndolo en el momento decisivo a contribuir a la erección de una máquina letal. Esta máquina schmittiana funciona expandiendo el umbral de la excepción, la nuda vida y la gloria al punto de hacer coincidir palmo a palmo lo político con lo impolítico, el derecho con la vida, el nomos con la anomia, la potestas con la auctoritas y el gobierno con la acclamatio. En suma, si nuestra modernidad política viene definida por el hecho de que la excepción que ha convertido en la regla, debemos concluir que Carl Schmitt es el representante eminente de la teoría política moderna (AGAMBEN, 2005, p. 2324).

\section{LA BIPOLARIDAD DE LA FORMA POLÍTICA SCHMITTIANA}

Debemos ahora preguntarnos si la teoría política de Schmitt se aviene al esquema desplegado en Homo sacer. De ser así, la máquina letal descrita por Agamben debería reflejar el legado schmittiano en sus rasgos más sistemáticos. En este sentido, interesa en lo que sigue inquirir cómo funciona la máquina política schmittiana.

La primera objeción al respecto es sugerida por Hugo Herrera, al recordar la crítica sistemática que Schmitt sostuvo ante el avance de la racionalidad técnica. Con esto en mente, resulta difícil reducir la teoría política schmittiana a una máquina o mecanismo (HERRERA, 2014, p. 178). Schmitt no niega que el oficio político conste de un momento técnico, vinculado a la manipulación de los factores de poder, pero su obra está orientada a postular que la política es irreductible a esa racionalidad instrumental.

Su ensayo Catolicismo romano y forma política apunta precisamente a caracterizar aquel sentido eminente de la política e irreductible a la técnica. Para ello, Schmitt se detiene en el desarrollo institucional de la Iglesia católica. En ese texto de 1923, sostiene Schmitt que, si bien la Iglesia no se aviene a la racionalidad instrumental predominante, eso no implica que ella carezca de toda racionalidad. Al contrario: mientras el racionalismo de la técnica se limita a los medios o 
instrumentos, el racionalismo católico apunta en cambio a los fines. Esto implica que la política no solo se relaciona con una técnica que persigue el poder sino también con una ética que persigue ideas. La política no puede reducirse al cálculo de conveniencias y a la mera comisión de intereses, sino que en ella entran también en juego convicciones y compromisos éticos. En esta línea, sostiene David Pan que Schmitt "no desatiende el análisis del poder, sino que más bien construye una teoría unificada del derecho, que entiende al poder y a la justicia como dos conceptos siempre vinculados" (PAN, 2009, p. 53). Compendia Schmitt:

\footnotetext{
Ningún sistema político puede perdurar una sola generación valiéndose simplemente de la técnica del mantenimiento del poder. La Idea es parte de lo Político, porque no hay política sin autoridad y no hay autoridad sin ethos de la convicción (SCHMITT, 1984, p. 45).
}

En este marco, Schmitt enfatiza el sentido eminentemente público de la representación política, que no consiste en una comisión de intereses o una mera gestión privada [Vertretung], sino que apunta a presentificar o personificar la idea [Repräsentation]. La autoridad que reviste el sacerdote no deriva de la representación de los intereses materiales de su grey, sino del hecho de ser vicario de Dios en la tierra. En esta línea, Schmitt enfatiza el carácter eminentemente personal de la representación: las ideas solo pueden representarse cuando se encarnan en la persona de la autoridad.

De este modo, Schmitt descubre en el racionalismo jurídico-institucional de la Iglesia católica la "complexio oppositorum" que define toda forma política (SCHMITT, 1984, p. 12). Concretamente, la forma política articula los polos opuestos de la técnica y la ética, del oficio y el sacerdocio, del poder y la autoridad. En esta composición de opuestos se encuentra la singular bipolaridad del pensamiento schmittiano.

Ahora bien, los polos de la auctoritas y la potestas reaparecen en su Teoría de la constitución, de 1928. Allí se sugiere la correspondencia de la auctoritas con el principio jurídico-formal de la representación y de la potestas con el principio de la identidad (SCHMITT, 2003, p. 76n.). Toda forma política se define a partir de una combinación de ambos polos opuestos: mientras la identidad remite a los rasgos inmediatos y reales que hacen a la homogeneidad del pueblo, la representación 
consiste en la postulación del principio espiritual que da forma a su existencia política (BIALAKOWSKY, 2011, p. 290-292). Si bien Schmitt sostiene que los dos principios son igualmente imprescindibles, todo su énfasis está puesto en el principio formador de la representación. Es que, para Schmitt, un pueblo homogéneo pero carente de representación constituiría una magnitud empírica sin existencia política. Para que un pueblo adquiera existencia política, debe darse una forma; y toda forma depende de una idea que funja de principio espiritual: "la idea de la representación se basa en que un pueblo existente como unidad política tiene una intensiva especie de ser, alta y elevada, frente a la existencia natural de cualquier grupo de humanos que vivan juntos" (SCHMITT, 2003, p. 210). Un pueblo sin representación, esto es, un pueblo carente de una idea elevada que dé forma política a su unidad, resulta un mero agregado de individuos con rasgos económicos, culturales o naturales más o menos homogéneos, pero carente de toda existencia política (SCHMITT, 2003, p. 212-215).

Por lo dicho hasta aquí, es posible identificar en las reflexiones schmittianas la postulación de una forma política bipolar, que articula poder y autoridad, identidad y representación. En este punto, es necesario considerar un episodio de singular importancia en la obra de Schmitt, donde los términos de autoridad y poder reciben su tratamiento más articulado y sistemático. Se trata del fragmento conocido como el Hobbes-Kristall, publicado en una extensa nota al pie que aparece en la edición de 1963 de El concepto de lo político. Schmitt presenta esta nota al pie como "el fruto del trabajo de toda una vida". Si bien allí se ofrece una reconstrucción de la arquitectura del sistema político presentado en el Leviatán, Schmitt aclara que la relevancia de este hallazgo va más allá del interés puntual por la obra de Hobbes y apunta a dar cuenta de un tema político superlativo. Schmitt presenta el cristal de Hobbes a partir del siguiente diagrama (SCHMITT, 2009, p. 113-114): 
Parte superior

Abierta a la trascendencia

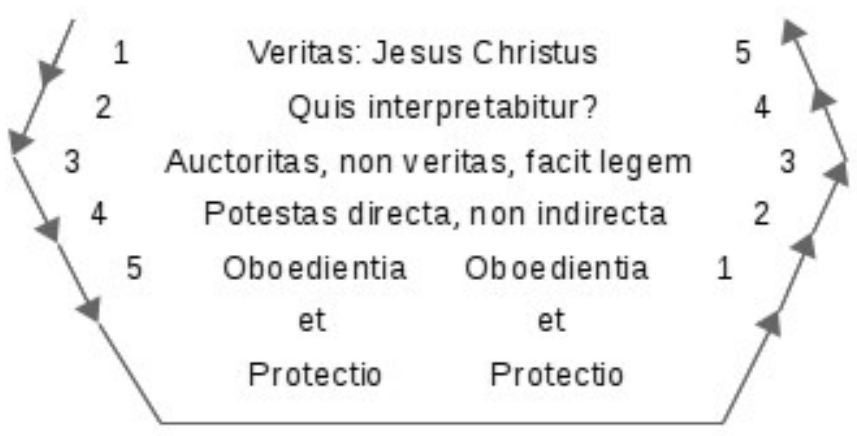

Parte inferior cerrada:

Sistema de las necesidades

De manera sugerente, la explicación de Schmitt comienza por la cúspide, sosteniendo que el postulado hobbesiano "Jesús es el Cristo" constituye la piedra angular que mantiene en pie el conjunto del edificio. De este modo, Schmitt identifica que el sistema de Hobbes se encuentra abierto a la trascendencia, es decir, que todo el edificio remata en una idea trascendente que constituye una verdad de fe pública. Agrega Schmitt que Hobbes es consciente de que la verdad de Jesucristo exige ser interpretada y que los conflictos en torno a esa interpretación han conducido a las guerras civiles religiosas. De allí que se derive por necesidad la pregunta de quién es el que interpreta esta verdad: “¿Quién decide cuál es el verdadero cristianismo? Éste es el inevitable Quis interpretabitur? y el incesante Quis judicabit?". Precisamente, quien interpreta la verdad, trasponiendo la idea al orden jurídico, es la autoridad. De allí se deriva la proposición "Autoritas, non veritas, facit legem". Schmitt prosigue advirtiendo que esta verdad plasmada en el orden jurídico no se realiza sola, sino que necesita instrucciones y mandatos que la hagan efectiva. Estas instrucciones y mandatos proceden de una potestas directa que, a diferencia de los poderes indirectos, logra concitar la obediencia porque está en condiciones de proteger a quienes la obedecen. Compendia Schmitt que, "de este modo, se produce una serie vertical, de arriba a abajo, que se extiende desde la verdad del culto público hasta la obediencia y protección del individuo" (SCHMITT, 2009, p. 113).

Schmitt muestra asimismo que este edificio puede ser recorrido de manera ascendente. En tal caso, el punto de partida viene dado por el afán de seguridad que 
experimenta cada individuo en el estado natural de desprotección. Sobre la base del sistema de las necesidades, se traba una relación entre los individuos y el poder, fundada en el intercambio de obediencia y protección. Ahora bien, el poder directo, que concita obediencia porque está en condiciones de proteger, solo puede mantener su promesa de seguridad allí donde la ley no está desgarrada por las disputas en lo relativo a la verdad. La paz civil exige que sea la autoridad quien determine el derecho. Es así que la autoridad realiza el derecho a partir de la apelación a una idea trascendente que da forma a la unidad política. En el caso de Hobbes, la idea trascendente es la verdad del Jesús-Cristo.

Llegado a este punto, sugiere Schmitt que la verdad postulada por Hobbes corresponde a la situación concreta de la Inglaterra de su época. Esto implica que otros pueblos podrían concebir formulaciones alternativas, más adecuadas a su situación concreta. En tal caso, la verdad de fe pública que provee la piedra angular del edificio político bien podría ser "Alá es grande", "Libertad, igualdad, fraternidad" o "A cada uno según sus méritos".

Como quiera sea, lo cierto es que Schmitt compone autoridad y poder en un edificio que se articula en dos mitades simétricas, cada una necesitada de la otra. Por un lado, en la parte inferior de la estructura, un agregado de individuos que se identifican en algunos rasgos comunes encuentran protección allí donde instituyen un poder común en condición de protegerlos. En la parte superior de la estructura, ese pueblo adquiere existencia política solo gracias a la postulación de una idea trascendente que es representada por la autoridad.

Ambas mitades, la infraestructura de la potestas y la superestructura de la auctoritas, se articulan en un doble movimiento. Por un lado, un movimiento ascendente, de politización del pueblo, que conduce del mero interés por la subsistencia a la presentificación de una idea elevada de ser que le da forma a la unidad política. Por otro lado, un movimiento descendente, de neutralización de los conflictos en torno a la verdad pública, gracias a la realización de la idea en el derecho y a la realización del derecho en la situación concreta. En este doble movimiento, autoridad y poder se distinguen y se reclaman de manera incesante.

Así queda presentado el edificio sistemático de la política hobbesiana y, con él, la forma política de la moderna estatalidad, tal como Schmitt la concibe. Se trata de una complexio oppositorum de poder y autoridad, de identidad y representación, 
de politización y neutralización, de intereses materiales e ideas configuradoras. Esto nos permite captar la singular bipolaridad de la forma política schmittiana. En este marco, debería resultar más comprensible el entusiasta apoyo que Schmitt tributó al nacionalsocialismo en ocasión de su ascenso. Veamos si efectivamente es así.

\section{LA ESTRUCTURA TRIÁDICA DE LA UNIDAD POLÍTICA}

Con seguridad, el aporte más resonante y pleno de consecuencias teóricas que Schmitt realizó al nacionalsocialismo está contenido en sus reflexiones de 1933 sobre la tríada Estado, movimiento, pueblo. Allí, Schmitt provee una caracterización del derecho público alemán adecuada a las transformaciones recientemente efectuadas por la revolución legal hitleriana. En vista de los desarrollos schmittianos precedentes, esta caracterización resulta llamativamente novedosa. Lo primero que salta a la vista, desde el subtítulo mismo del tratado, es que la unidad política no remite a una estructura bipolar sino triádica. Precisamente, el tercer apartado de este texto está dedicado a denunciar la obsolescencia de los dualismos característicos de la democracia liberal. Según informa Schmitt, antítesis liberales como las de "derecho y poder" o "espíritu y poder" han sido en todo superadas por la emergencia del nuevo orden jurídico (SCHMITT, 1933, p. 22).

Schmitt celebra que el derecho público alemán se erija finalmente sobre "sus propios cimientos". Precisamente, la ley fundamental del derecho público emergente consiste en dar prioridad a una figura específicamente alemana, que es la de la Führung (SCHMITT, 1933, p. 5, 9). Schmitt se preocupa por resaltar la singularidad y la esencia alemana de esta figura, distinguiéndola de toda otra forma de conducción política. Resulta llamativo, en este punto, que el Führertum elogiado por Schmitt resulte irreductible a la forma política del catolicismo romano. Recuerda Schmitt:

\footnotetext{
La Iglesia católica romana ha moldeado su poder de mando sobre los creyentes, introduciendo la imagen del pastor y del rebaño en un pensamiento teológico-dogmático. Es de la esencia de esta imagen el que el pastor permanezca en una trascendencia absoluta respecto del rebaño (SCHMITT, 1933, p. 41).
}

Contra esto, Schmitt enfatiza que el Führer no trasciende a su pueblo, sino que traba con él un vínculo directo, sin mediaciones ni representaciones. Completa 
Schmitt que esta figura "no proviene de alegorías ni representaciones barrocas, ni de una idée générale cartesiana" (SCHMITT, 1933, p. 42). Así, esta inédita forma política nada le debe al pastorado de la Iglesia de Roma ni al sistema barroco del Leviatán de Hobbes. Se trata de "un concepto de actualidad inmediata y presencia real. Por esta razón, incluye como requisito positivo una inmediata identidad de especie [Artgleichheit] entre el conductor y su séquito" (SCHMITT, 1933, p. 42).

De este modo, la revolución legal de Hitler concitará una reconfiguración profunda de la estructura política schmittiana. Si hasta entonces la forma política era definida por la articulación de autoridad y poder o de representación e identidad, con Estado, movimiento, pueblo asistimos al rechazo de toda trascendencia y a la erección de una forma política resuelta en la inmediatez de la identidad racial. Es así que el énfasis schmittiano en la representación como principio formativo de la unidad política desaparece para dar lugar a una absolutización del principio de identidad. De igual modo, el énfasis en la autoridad como presentificación de la idea desaparece para dar lugar a una absolutización del poder del conductor. Dejando atrás la distinción entre auctoritas y potestas, sentencia Schmitt que "lo vivo no puede legitimarse en lo muerto y la fuerza tampoco precisa legitimarse en la falta de fuerza" (SCHMITT, 1933, p. 8). En este marco de extrapolación del principio de identidad, Schmitt formula la estructura trimembre de la unidad política:

Cada una de las tres palabras Estado, Movimiento, Pueblo, puede por sí sola portar la totalidad de la unidad política. No obstante, cada una de ellas indica una parte especial y un elemento específico de esta totalidad. Así, el Estado en sentido propio puede considerarse como la parte político-estática; el movimiento, como el elemento político-dinámico; y el pueblo, como el lado impolítico que crece bajo la protección y a la sombra de las decisiones políticas (SCHMITT, 1933, p. 12).

En esta estructura trimembre, el elemento determinante viene dado por el movimiento que el Führer imprime sobre el conjunto de la unidad política. Precisamente, la determinación de lo político y lo apolítico $-\mathrm{y}$, por ende, de aquello que pertenece al Estado y aquello que pertenece al pueblo- resulta de la conducción política y de las decisiones políticas del movimiento (GALLI, 1996, p. 859; Cf. VAZCURADO, 2014, p. 285-301). Schmitt sostiene que toda arbitrariedad y tiranía queda conjurada en virtud del principio de identidad racial que vincula personalmente al Führer con el pueblo y con el Estado. 
También "el funcionario es un camarada del pueblo en una unidad política basada en la igualdad de especie [Artgleichheit]" (SCHMITT, 1933, p. 17). La identidad racial constituye de este modo la garantía de objetividad e imparcialidad de funcionarios y jueces. Corresponde a ellos interpretar aquellos conceptos jurídicos indeterminados que han sido incorporados al derecho alemán y que remiten al orden concreto. En última instancia, todo depende de "la especie y tipo de nuestros jueces y funcionarios. La pregunta 'quis judicabit' nunca ha revestido importancia tan crucial como hoy" (SCHMITT, 1933, p. 44).

Si revisamos estos sorprendentes desarrollos a la luz de la teoría política schmittiana presentada en el apartado anterior, es posible describir el desplazamiento operado por Schmitt en 1933 en los términos de un repliegue que hace coincidir los extremos de la estructura política. Concretamente, la idea trascendente se sobrepone al sistema de las necesidades, la verdad de fe pública coincide con la protección de la especie y la representación se diluye en la mera identidad racial. De este modo, la complexio oppositorum que define la forma política queda abolida. En su reemplazo emerge una nueva forma, caracterizada por la apretada identidad de todos los términos intervinientes.

Si retomamos el Hobbes-Kristall presentado más arriba, la operación teórica que realiza Schmitt en 1933 equivaldría a plegar el cristal por su línea central, haciendo coincidir la parte superior del edificio con la inferior. De ser así, la estructura triádica de la unidad política podría graficarse del modo siguiente:

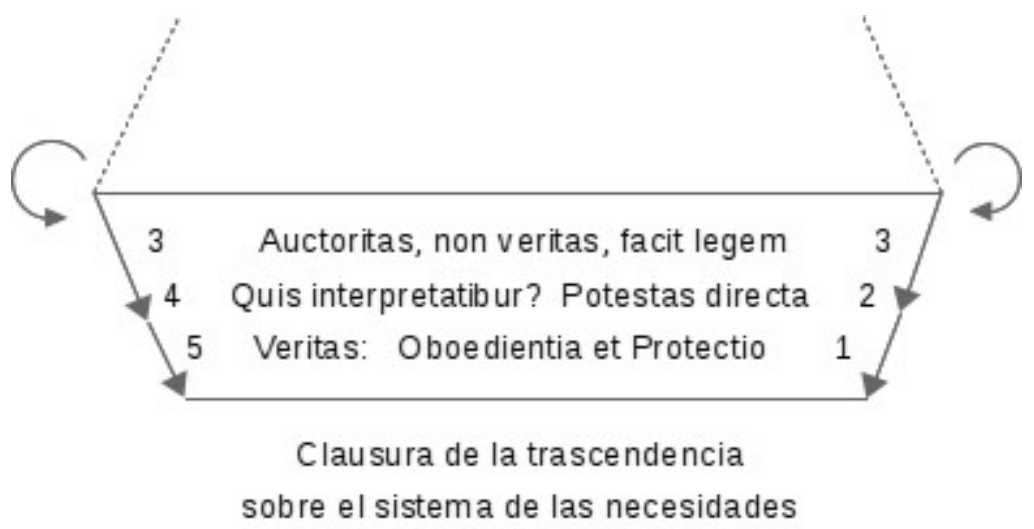

Con la obturación de toda trascendencia, la mera existencia del pueblo alemán se sobrepone a toda idea formadora de la unidad política. Esto hace que la 
verdad de fe pública coincida con la protección del pueblo, definido en términos de su identidad racial. Sobre este pueblo se monta una estructura estatal reducida a su faz puramente técnica, de funcionarios y jueces que, más que aplicar la ley, interpretan los conceptos jurídicos indeterminados que remiten al orden concreto (LALEFF ILIEFF, 2015, pp. 201-202). La estructura se remata con el momento político-dinámico del movimiento que imprime el Führer. La figura de Hitler no remite, sin embargo, a una autoridad representativa: no se trata aquí de presentificar una idea trascendente, sino de manifestar la identidad inmediata y directa del pastor con su rebaño. Sin deudas con el pasado, a distancia de todo legado teológico o barroco, la teoría estatal schmittiana introduce en 1933 una forma política decididamente insólita.

\section{ESCATOLOGÍA O KATECHON}

Ha llegado el momento de recapitular y concluir. Nuestro punto de partida estuvo dado por la constatación superficial de una analogía estructural en los modos en que Schmitt y Agamben conciben la política. Concretamente, la estructura política consiste en ambos casos en la conjunción de dos términos mutuamente inasimilables pero igualmente imprescindibles. Esta constatación primera de la común bipolaridad de ambas teorías reclamaba sin embargo un abordaje más pormenorizado y cabal.

La clave para embarcarnos en la máquina política agambeniana fue provista por el último volumen de la saga Homo sacer. Allí, Agamben recopila los resultados de su arqueología de la política occidental, identificando que en todas sus investigaciones una misma estructura se encontraba en obra. Concretamente, el arcano de la política occidental consiste en el establecimiento de una cesura en la experiencia fáctica, que permite producir una interioridad expulsando lo exteriorizado al fondo o al origen, y recapturándolo en la forma de fundamento. De este modo, la máquina política funciona produciendo los polos de lo político y lo impolítico, y recapturando lo excluido en la forma de la excepción.

En su arqueología de la política, Homo sacer se remonta hasta la antigüedad griega. En algún sentido, Agamben identifica que la Grecia clásica encontró la manera de equilibrar las tensiones de la máquina política, cuanto menos de un modo 
precario e incierto (AGAMBEN, 2015, p. 31). En la modernidad, en cambio, esa estructura bipolar se vuelve profundamente inestable, a punto tal que la excepción finalmente resulta desbocada y la precaria estructura política se corrompe, dando lugar a una máquina letal.

En este marco, la importancia que para Agamben tiene la teoría política schmittiana es también doble: si, por un lado, Schmitt se abocó a la comprensión de la estructura política occidental con una penetración sin igual; por otro lado, lo cierto es que, en el momento decisivo, contribuyó como pocos al descalabro que condujo a la catástrofe moderna (TAUB, 2013, p. 89).

Habiendo restituido la estructura política presentada en Homo sacer, nos interesó censar la obra de Schmitt a efectos de comprender la singular bipolaridad que caracteriza su propia comprensión de la forma política y ponderar seguidamente la razonabilidad de las acusaciones agambenianas. La genealogía schmittiana de la política no nos condujo a Atenas, sino a Roma. Precisamente, Schmitt entronca la forma política en el racionalismo jurídico-institucional de la Iglesia católica, caracterizado por la composición de los opuestos de la auctoritas y la potestas. Será Thomas Hobbes quien trasponga esa estructura bipolar al estado moderno, articulando el poder que protege con la autoridad que representa. Al igual que Agamben, pero en virtud de otras razones, Schmitt identifica que la forma política cae en desgracia durante el siglo XX. Sus críticas más recurrentes apuntan contra la fe en la técnica, que promete paz y seguridad, reduciendo la forma política al sistema de las necesidades. De este modo, liberales y comunistas demuestran para Schmitt una oculta solidaridad en la postulación de una política resuelta en la inmanencia y reñida con toda autoridad. Lo que Schmitt no explicita es que también él, en el momento decisivo, se arrojó a una política de la pura inmanencia, trocando la representación de la idea por la mera identidad de estirpe, y la dignidad personal de la autoridad por la fuerza inmediata del conductor.

A pocos años de concluida la catástrofe alemana, Schmitt registró en sus cuadernos personales algunas reflexiones sobre la utopía moderna de la plena securitas: esa falsa promesa de la técnica que constituye un engaño ampliamente expandido en nuestro tiempo. Señala Schmitt que, ante el sostenido incumplimiento de esa promesa, no queda más que descargar en chivos expiatorios, sean "los capitalistas, los judíos, los jesuitas o Hitler" (SCHMITT, 2015, p. 71). En lo que no 
constituye siquiera un principio de autocrítica, Schmitt logra divisar la magnitud del peligro implicado en una política exclusivamente abocada la protección de la vida.

El último documento del expediente Schmitt/Agamben que nos queda por abordar remite al problema del tiempo histórico. A efectos de comprender el tiempo que nos es contemporáneo, Schmitt propone recurrir a la imagen cristiana de la historia que surge de la segunda carta de Pablo a los tesalonicenses. Allí Pablo advierte que el juicio final que marcará el fin de los tiempos será precedido por la manifestación del anticristo, del hombre de la anomia. Si bien la anomia ya se encuentra en acto, también actúa una fuerza que la retiene para que se manifieste a su debido tiempo. Schmitt se interesa en esta fuerza que retiene la anomia (to katéchon). En ella identifica la función del imperium durante la Respublica Christiana (SCHMITT, 1974, p. 28-32). De igual modo, si el estado moderno constituye una trasposición de la forma católica, es precisamente porque él también dispone una fuerza catecóntica que demora la manifestación del anticristo. Estas fuerzas en lucha son magistralmente desplegadas por la iconología de Thomas Hobbes, al asignar al Estado la figura de un Leviatán que lucha contra el demoníaco Behemoth (SCHMITT, 2012).

Agamben resiste esta interpretación catecóntica de la estatalidad moderna. Lo hace señalando que Hobbes era absolutamente consciente de que la imagen del Leviatán había sido durante mucho tiempo, y todavía en su época, sinónimo del anticristo. Agamben argumenta que, al recurrir al monstruo bíblico, Hobbes inscribió la estatalidad moderna en una perspectiva decididamente escatológica. De este modo, en la perspectiva agambeniana, el estado moderno conjuga tanto la fuerza que detiene como la manifestación de la anomia (AGAMBEN, 2015, p. 75-77; 2000, pp. 98-105). Recientemente Fabián Ludueña Romandini se ha detenido en esta querella, rastreando con singular erudición las interpretaciones patrísticas y contemporáneas del pasaje paulino. No podemos aquí más que remitir a aquella sutil reconstrucción (LUDUEÑA ROMANDINI, 2016, p. 4-30).

En lo que a nosotros respecta, interesa señalar que esta querella teológica tiene un correlato evidente tanto en términos de la comprensión teórica del Estado como en términos de la orientación de una práctica política consecuente. De lo que aquí se trata es de saber si la deriva anómica y totalitaria es inherente al moderno Leviatán; o si es solo en virtud de la mutilación de su forma política que el Estado 
puede volverse promotor de la catástrofe. En el primer caso, la estatalidad representa aquello que debe ser depuesto sin ambages. En el segundo, cabrá pensar que allí donde está el peligro, crece también lo que salva. De la respuesta a estas interrogantes depende la orientación de nuestros temores y esperanzas.

\section{REFERENCIAS}

AGAMBEN, Giorgio. Homo sacer, I. Il potere sovrano e la nuda vita. Turín. Einaudi, 1995.

. Il tempo che resta. Un commento alla Lettera ai Romani. Turín. Bollati Bolinghieri, 2000.

Stato di eccezione. Homo sacer, II, 1. Turín. Bollati Bolinghieri, 2003.

. Entrevista de Flavia Costa. In: AGAMBEN, Giorgio. Estado de excepción. Buenos Aires. Adriana Hidalgo, 2004.

. Introduzione. In: AGAMBEN, Giorgio (Cur.). Carl Schmitt: Un giurista davanti a se stesso. Saggi e interviste. Vicenza. Neri Pozza, 2005, pp. 7-28.

. II regno e la gloria. Per una genealogia teologica dell'economia e del governo. Homo sacer, II, 2. Vicenza. Neri Pozza, 2007.

. L'uso dei corpi. Homo sacer, IV, 2. Vicenza. Neri Pozza, 2014.

Stasis. La guerra civile come paradigma politico. Homo sacer, II, 2. Turín. Bollati Bolinghieri, 2015.

BIALAKOWSKY, Guillermo. Tiempo y representación. La crítica impolítica italiana al pensamiento de Carl Schmitt. Instantes y Azares. Escrituras Nietzscheanas, Buenos Aires, n. 9, p. 289-300, 2011.

CAVALLETTI, Andrea. El filósofo inoperoso. Deus Mortalis, Buenos Aires, n. 9, p. $51-71,2010$.

GALLI, Carlo. Genealogia della politica. Carl Schmitt e la crisi del pensiero moderno, Boloña, II Mulino, 1996.

HERRERA, Hugo. Racionalidad técnica y excepción. La crítica de Giorgio Agamben a la doctrina del estado de excepción de Carl Schmitt y sus alcances. Anales del Seminario de Historia de la Filosofía, Madrid, v. 31, n. 1, p. 177-192, 2014. 
LALEFF ILIEFF, Ricardo. Herederos de 1848. Un contrapunto en torno al pensamiento político de entreguerras de Antonio Gramsci y Carl Schmitt. 2015. 282 f. Tesis (doctoral) Universidad de Buenos Aires, Buenos Aires, 2015.

LALEFF ILIEFF, Ricardo. Politización y despolitización en el pensamiento de Carl Schmitt. Ágora filosófica, Pernambuco, a. 16, n. 2, p. 63-75, jul/dez. 2016-2.

LUDUEÑA ROMANDINI, Fabián. EI mesianismo agambeniano entre Cacciari Y Žižek. Una arqueología del Katéchon. Profanações, Santa Catarina, a. 3, n. 2, p. 430, jul./dez. 2016.

MOYANO, Manuel. Ontología de la inoperancia. In: BISSET, Emmanuel y FARRÁN, Roque (Eds.) Ontologías políticas. Buenos Aires. Imago Mundi, 2011.

MOYANO, Manuel. Agamben y el Estado. Soberanía, gobierno, espectáculo: la "vida desnuda”. In: BISET, Emannuel y FARRÁN, Roque (Comps.) Estado: Perspectivas postfundacionales. Buenos Aires. Prometeo, 2017.

PAN, David. Against Biopolitics: Walter Benjamin, Carl Schmitt, and Giorgio Agamben on Political Sovereignty and Symbolic Order. The German Quarterly, Nueva Jersey, v. 82 , n. 1, p. 42-62, 2009.

PRÓSPERI, Germán. La máquina elíptica de Giorgio Agamben. Profanações, Santa Catarina, a. 2, n. 2, pp. 62-83, jul./dez. 2015.

RAFFIN, Marcelo. Excepción, nomos y teología política: la recepción agambeniana del pensamiento de Schmitt. In: Jornadas Actualidad de Carl Schmitt a 30 años de su muerte. Universidad de Buenos Aires, 18 de noviembre de 2015. Disponible en: https://www.youtube.com/watch?v=VUd1sdRzGhs\&t=4737s.

SCHMITT, Carl. Staat, Bewegung, Volk. Die Dreigliederung der politischen Einheit. Hamburgo. Hanseatische Verlagsanstatl, 1993.

SCHMITT, Carl. Der Nomos der Erde. Im Völkerrecht des Jus Publicum Europaeum. Berlín. Duncker \& Humblot, 1974.

SCHMITT, Carl. Römischer Katholizismus und politische Form. Stuttgart. KlettCotta, 1984.

SCHMITT, Carl. Verfassungslehre. Berlín. Duncker \& Humblot, 2003.

SCHMITT, Carl. Politische Theologie. Vier Kapitel zur Lehre von der Souveränität. Berlín. Duncker \& Humblot, 2004.

SCHMITT, Carl. Der Begriff des Politischen. Text von 1932 mit einem Vorwort und drei Corollarien. Berlín: Duncker \& Humblot, 2009.

SCHMITT, Carl. Der Leviathan in der Staatslehre des Thomas Hobbes. Sinn und Fehlschlag eines politischen Symbols. Stuttgart: Klett-Cotta, 2012. 
SCHMITT, Carl. Glossarium. Aufzeichnungen aus den Jahren 1947 bis 1958. Berlín. Duncker \& Humblot, 2015.

TACCETTA, Natalia. Agamben y lo político. Buenos Aires, Prometeo, 2012.

TAUB, Emmanuel. Del paradigma de la excepción a la teología política. Los fundamentos schmittianos en Giorgio Agamben. Studia Politicae, Córdoba, n. 28, p. 77-98, 2013.

VAZ-CURADO, Danilo. A constituição ambígua do político em Staat, Bewegung, Volk de Carl Schmitt. Problemata, Paraíba, v. 5, n. 2, p. 285-301, 2014.

Artigo recebido em: 23/03/2017

Artigo aprovado em: 15/11/2017

Artigo publicado em: 18/12/2017 